\title{
A new type of intuitionistic fuzzy modal operators over intuitionistic fuzzy pairs
}

\author{
Krassimir T. Atanassov \\ Department of Bioinformatics and Mathematical Modelling, \\ Institute of Biophysics and Biomedical Engineering, \\ Bulgarian Academy of Sciences \\ 105 Acad. G. Bonchev Str., 1113 Sofia, Bulgaria \\ e-mail: kratebas.bg
}

Received: 12 October 2021

Accepted: 30 November 2021

\begin{abstract}
In the paper, two intuitionistic fuzzy modal operators from a new type are introduced over intuitionistic fuzzy pairs. Some of the basic properties of the new operators are formulated and checked.
\end{abstract}

Keywords: Intuitionistic fuzzy modal operator, Intuitionistic fuzzy pair.

2020 Mathematics Subject Classification: 03E72.

\section{Introduction}

The concept of an Intuitionistic Fuzzy Pair (IFP) was introduced in [4] independently with Z. Xu's paper [6], where he called the same object an intuitionistic fuzzy value. In a series of papers, a lot of operations, relations and operators are defined over IFPs (see, e.g., [2]). A part of them are given in Section 2 and they are used in Section 3, where two modal operators from a new type are introduced and some of their basic properties are given.

\section{Preliminaries}

In [4], the pair $\langle a, b\rangle$ so that $a, b, a+b \in[0,1]$ is called an IFP. For two pairs $x=\langle a, b\rangle$ and $y=\langle c, d\rangle$, the following relations, operations and operators (and a lot of others) are introduced: 


$$
\begin{aligned}
& x \leq y \text { iff } a \leq c \text { and } b \geq d, \\
& x=y \text { iff } x \leq y \text { and } y \leq x, \\
& x \wedge y=\langle\min (a, c), \max (b, d)\rangle, \\
& x \vee y=\langle\max (a, c), \min (b, d)\rangle, \\
& x+y=\langle a+c-a c, b d\rangle \text {, } \\
& x . y=\langle a c, b+d-b d\rangle, \\
& x @ y=\left\langle\frac{a+c}{2}, \frac{b+d}{2}\right\rangle, \\
& \neg x=\langle b, a\rangle ; \\
& \square x=\langle a, 1-a\rangle, \\
& \diamond x=\langle 1-b, b\rangle, \\
& D_{\alpha} x=\langle a+\alpha(1-a-b), b+(1-\alpha)(1-a-b)\rangle, \\
& F_{\alpha, \beta} x=\langle a+\alpha(1-a-b), b+\beta(1-a-b)\rangle, \\
& G_{\alpha, \beta} x=\langle\alpha a, \beta b\rangle \text {, } \\
& H_{\alpha, \beta} x=\langle\alpha a, b+\beta(1-a-b)\rangle \text {, } \\
& H_{\alpha, \beta}^{*} x=\langle\alpha a, b+\beta(1-\alpha a-b)\rangle \text {, } \\
& J_{\alpha, \beta} x=\langle a+\alpha(1-a-b), \beta b\rangle, \\
& J_{\alpha, \beta}^{*} x=\langle a+\alpha(1-a-\beta b), \beta b\rangle ; \\
& \bullet_{\alpha, \beta, \gamma, \delta} x=\langle\alpha a+\gamma, \beta b+\delta\rangle \text {, } \\
& \text { where } \alpha, \beta, \gamma, \delta \in[0,1] \text { and } \max (\alpha, \beta)+\gamma+\delta \leq 1 \text {, } \\
& \square_{\alpha, \beta, \gamma, \delta, \varepsilon, \zeta} x=\langle\alpha a-\varepsilon b+\gamma, \beta b-\zeta a+\delta\rangle \text {, } \\
& \text { where } \alpha, \beta, \gamma, \delta, \varepsilon, \zeta \in[0,1], \max (\alpha-\zeta, \beta-\varepsilon)+\gamma+\delta \leq 1 \text {, } \\
& \text { and } \min (\alpha-\zeta, \beta-\varepsilon)+\gamma+\delta \geq 0 \text {, } \\
& \otimes_{\alpha, \beta, \gamma, \delta} x=\langle\alpha a+\gamma b, \beta a+\delta b\rangle \text {, } \\
& \text { where } \alpha, \beta, \gamma, \delta \in[0,1] \text { and } \alpha+\beta \leq 1, \gamma+\delta \leq 1 \text {. }
\end{aligned}
$$

\section{Main results}

Everywhere below we will use:

$$
\begin{aligned}
& x=\langle a, b\rangle, \\
& y=\langle c, d\rangle .
\end{aligned}
$$

Let us define the following two new operators:

$$
\begin{aligned}
& \triangle x=\triangle\langle a, b\rangle=\langle a+b, 0\rangle, \\
& \nabla x=\nabla\langle a, b\rangle=\langle 0, a+b\rangle .
\end{aligned}
$$

The geometrical interpretation of both operators is shown on Fig. 1. 


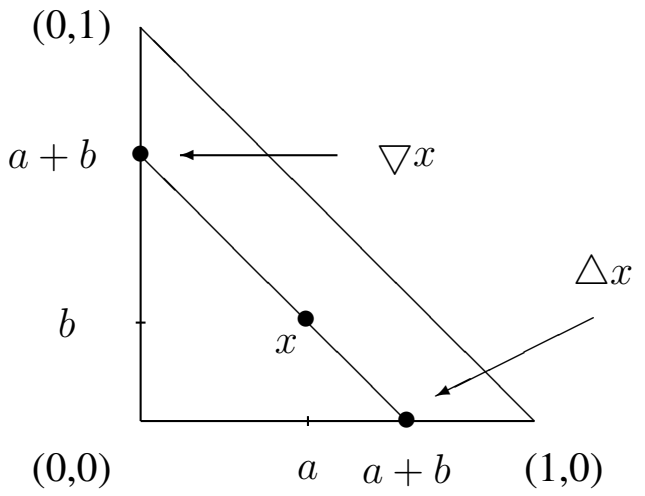

Figure 1. Geometrical interpretation of an element of an IFS.

We see immediately that

$$
\begin{gathered}
\triangle\langle 1,0\rangle=\triangle\langle 0,1\rangle=\langle 1,0\rangle, \\
\nabla\langle 1,0\rangle=\nabla\langle 0,1\rangle=\langle 0,1\rangle, \\
\nabla x \leq x \leq \triangle x .
\end{gathered}
$$

The new operators have the following properties.

Proposition 1. For each IFP $x$ :

(a) $\triangle \nabla x=\triangle x$,

(b) $\nabla \triangle x=\nabla x$.

Proof. (a) Let $x$ be an IFP. Then

$$
\triangle \nabla x=\triangle \nabla\langle a, b\rangle=\triangle\langle 0, a+b\rangle=\langle a+b, 0\rangle=\triangle x
$$

(b) The assertion is proved analogically.

Therefore, there is an important difference in the behaviour of the new modal operators and the intuitionistic fuzzy forms of the classical modal operators, that satisfy equalities $\square \diamond x=\diamond x$ and $\diamond \square x=\square x(\operatorname{cf}[2,5])$.

Between the two types of modal operators there are the following relationships.

Proposition 2. For each IFP $x$ :

(a) $\square \triangle x \leq \triangle \square x$,

(b) $\square \nabla x=\nabla \square x$,

(c) $\diamond \triangle x=\Delta \diamond x$,

(d) $\diamond \nabla x \geq \nabla \diamond x$. 
Proof. We check directly that:

$$
\begin{aligned}
& \square \triangle x=\square\langle a+b, 0\rangle=\langle a+b, 1-a-b\rangle \leq\langle 1,0\rangle=\triangle\langle a, 1-a\rangle=\triangle \square x, \\
& \square \nabla x=\square\langle 0, a+b\rangle=\langle 0,1\rangle=\nabla\langle a, 1-a\rangle=\nabla \square x, \\
& \diamond \triangle x=\diamond\langle a+b, 0\rangle=\langle 1,0\rangle=\triangle\langle a, 1-a\rangle=\triangle \square x, \\
& \diamond \nabla x=\diamond\langle 0, a+b\rangle=\langle 1-a-b, a+b\rangle \geq\langle 0,1\rangle=\nabla\langle 1-b, b\rangle=\nabla \diamond x .
\end{aligned}
$$

This completes the proof.

In [2] it is shown that operator $D_{\alpha}$ extends both operators $\square$ and $\diamond$ because for each IFP $x$ : $\square x=D_{0}$ and $\diamond x=D_{1} x$, while operator $F_{\alpha, \beta}$ extends operator $D_{\alpha}$, because for each IFP $x$ : $D_{\alpha}=f_{\alpha, 1-\alpha}$. Now, an extension of Proposition 2 has the following form.

Proposition 3. For each IFP $x$ and for every $\alpha, \beta \in[0,1]$ so that $a+b \in[0,1]$ :

(a) $D_{\alpha} \triangle x \leq \triangle D_{a l} x$,

(b) $D_{\alpha} \nabla x \geq \nabla D_{a l} x$,

(c) $F_{\alpha, \beta} \triangle x \leq \triangle F_{a l, \beta} x$,

(d) $F_{\alpha, \beta} \nabla x \geq \nabla F_{a l, \beta} x$.

More interesting is the case of the relationships between the new operators and operator $G_{\alpha, \beta}$. Proposition 4. For each IFP $x$ and for every $\alpha, \beta \in[0,1]$ :

(a) if $\alpha \leq \beta$, then

$$
\begin{aligned}
& G_{\alpha, \beta} \triangle x \leq \triangle G_{a l, \beta} x, \\
& G_{\alpha, \beta} \nabla x \geq \nabla G_{a l, \beta} x,
\end{aligned}
$$

(b) if $\alpha \geq \beta$, then

$$
\begin{aligned}
& G_{\alpha, \beta} \triangle x \geq \triangle G_{a l, \beta} x, \\
& G_{\alpha, \beta} \nabla x \leq \nabla G_{a l, \beta} x .
\end{aligned}
$$

Proposition 5. For each IFP $x$ and for every $\alpha, \beta \in[0,1]$ so that $a+b \in[0,1]$ :

(a) $H_{\alpha, \beta} \triangle x \leq \triangle H_{a l, \beta} x$,

(b) $H_{\alpha, \beta} \nabla x \geq \nabla H_{a l, \beta} x$,

(c) $J_{\alpha, \beta} \triangle x \geq \triangle J_{a l, \beta} x$,

(d) $J_{\alpha, \beta} \nabla x \leq \nabla J_{a l, \beta} x$,

(e) $H_{\alpha, \beta}^{*} \triangle x \leq \triangle H_{a l, \beta}^{*} x$,

(f) $H_{\alpha, \beta}^{*} \nabla x \geq \nabla H_{a l, \beta}^{*} x$, 
(g) $J_{\alpha, \beta}^{*} \triangle x \geq \triangle J_{a l, \beta}^{*} x$,

(h) $J_{\alpha, \beta}^{*} \nabla x \leq \nabla J_{a l, \beta}^{*} x$.

Proposition 6. For each IFP $x$ :

(a) $\neg \triangle \neg x=\nabla x$,

(b) $\neg \nabla \neg x=\triangle x$,

The relationships between the new operators and some of the operations over IFPs are the following.

Proposition 7. For every two IFPs $x$ and $y$ :

(a) $\triangle(x+y) \leq \triangle x+\triangle y$,

(b) $\triangle(x . y) \geq \triangle x \cdot \triangle y$,

(c) $\triangle(x @ y)=\triangle x @ \triangle y$,

(d) $\nabla(x+y) \geq \nabla x+\nabla y$,

(e) $\nabla(x . y) \leq \nabla x \cdot \nabla y$

(f) $\nabla(x @ y)=\nabla x @ \nabla y$.

\section{Conclusion}

The so introduced intuitionistic fuzzy modal operators over IFPs can be modified (and this will be an object of a nexr research of the author) for the case of Intuitionistic Fuzzy Sets (IFSs, see, e.g., [1]) and interval-valued IFSs (see, e.g., [3]).

The new operators can have application in different areas as Data Mining, Decision making, intercriteria analysis and others.

\section{Acknowledgement}

This research was funded by Bulgarian National Science Fund, grant number KP-06-N22/1/2018 "Theoretical research and applications of InterCriteria Analysis".

\section{References}

[1] Atanassov, K. (2012). On Intuitionistic Fuzzy Sets Theory. Berlin: Springer.

[2] Atanassov, K. (2017). Intuitionistic Fuzzy Logics. Cham: Springer. 
[3] Atanassov, K. (2020). Interval-Valued Intuitionistic Fuzzy Sets. Cham: Springer.

[4] Atanassov, K., Szmidt, E., \& Kacprzyk, J. (2013). On intuitionistic fuzzy pairs. Notes on Intuitionistic Fuzzy Sets, 19(3), 1-13.

[5] Feys, R. (1965). Modal logics. Paris: Gauthier-Villars.

[6] Xu, Z. (2007). Intuitionistic Fuzzy Aggregation Operators. IEEE Transactions on Fuzzy Systems, 15(6), 1179-1187. 